\title{
ROSENTHAL SETS AND THE RADON-NIKODYM PROPERTY
}

\author{
PATRICK N. DOWLING
}

(Received 5 April 1991; revised 24 July 1991)

Communicated by P. G. Dodds

\begin{abstract}
Let $X$ be a complex Banach space, $G$ a compact abelian metrizable group and $\Lambda$ a subset of $\widehat{G}$, the dual group of $G$. If $X$ has the Radon-Nikodym property and $L_{\Lambda}^{\infty}(G ; X)$ is separable, then $L_{\Lambda}^{\infty}(G, X)$ has the Radon-Nikodym property. One consequence of this is that $C_{\Lambda}(G, X)$ has the Radon-Nikodym property whenever $X$ has the Radon-Nikodym property and the Schur property and $\Lambda$ is a Rosenthal set. A partial stability property for products of Rosenthal sets is also obtained.
\end{abstract}

1991 Mathematics subject classification (Amer. Math. Soc.): 46 B 22, 43 A 46.

Keywords and phrases: Rosenthal sets, Radon-Nikodym property.

\section{Introduction}

For Banach spaces $X$ and $Y$ we denote by $\mathscr{L}(X, Y)$ the Banach space of all bounded linear operators from $X$ into $Y$. In this set-up, Pettis's last theorem states: if $\mathscr{L}\left(X, Y^{*}\right)$ is separable, then $\mathscr{L}\left(X, Y^{*}\right)$ has the RadonNikodym property [3, p. 165]. Notice that if $\mathscr{L}\left(X, Y^{*}\right)$ is separable, then $X^{*}$ and $Y^{*}$ are separable and so $X^{*}$ and $Y^{*}$ have the Radon-Nikodym property.

In this note, we will prove a result which is a variant of Pettis's last theorem. In our case, we will replace $X$ by a particular quotient of $L^{1}$ and $Y^{*}$ by an arbitrary Banach space with the Radon-Nikodym property. To be more specific, if $G$ is a compact abelian metrizable group and $\Lambda$ is a Rosenthal subset of $\widehat{G}$, then $L_{\Lambda}^{\infty}(G)$ is a separable dual space. If we

(C) 1993 Australian Mathematical Society $0263-6115 / 93 \$ A 2.00+0.00$ 
let $\Lambda^{\prime}=\{\gamma \in \widehat{G}: \bar{\gamma} \notin \Lambda\}$, then $L_{\Lambda}^{\infty}(G)$ is the dual of $L^{1}(G) / L_{\Lambda^{\prime}}^{1}(G)$. We will show that $\mathscr{L}\left(L^{1}(G) / L_{\Lambda^{\prime}}^{1}(G), X\right)$ has the Radon-Nikodym property whenever $X$ has the Radon-Nikodym property and $\mathscr{L}\left(L^{1}(G) / L_{\Lambda^{\prime}}^{1}(G), X\right)$ is separable. In fact, a close analysis of Lemma 4 of $[2$, p. 62] yields that proving the above result is equivalent to proving that $L_{\Lambda}^{\infty}(G, X)$ has the Radon-Nikodym property whenever $X$ has the Radon-Nikodym property and $L_{\Lambda}^{\infty}(G, X)$ is separable. This is the first result we prove in the next section.

One consequence of these results is that if $\Lambda$ is a Rosenthal subset of $\widehat{G}$ and $X$ is a complex Banach space with the Radon-Nikodym property and the Schur property, then $C_{\Lambda}(G, X)$ has the Radon-Nikodym property. It is unknown, at the moment, if the restriction of $X$ having the Schur property is necessary. Actually, a related question, which is also still unanswered, is the following: if $G_{1}$ and $G_{2}$ are two compact abelian metrizable groups and $\Lambda_{1}$ and $\Lambda_{2}$ are two Rosenthal subsets of $\widehat{G}_{1}$ and $\widehat{G}_{2}$, respectively, is $\Lambda_{1} \times \Lambda_{2}$ a Rosenthal subset of $\widehat{G}_{1} \times \widehat{G}_{2}$ ? However, we will be able to show that if $\Lambda_{1}$ is a Rosenthal set and $\Lambda_{2}$ is such that $L_{\Lambda_{2}}^{\infty}\left(G_{2}\right)$ has the Schur property, then $\Lambda_{1} \times \Lambda_{2}$ is a Rosenthal set. The question of whether the product of two Rosenthal sets is again a Rosenthal set was first raised by $F$. Lust-Piquard.

\section{The results}

Throughout this section, $G$ will denote a compact abelian metrizable group, $\mathscr{B}(G)$ denotes the $\sigma$-algebra of Borel subsets of $G$ and $\lambda$ is normalised Haar measure on $G$. The dual group of $G$ is denoted by $\widehat{G}$. If $f$ is a function defined on $G$ and $\gamma \in \widehat{G}$, we define $\hat{f}(\gamma)$ by

$$
\hat{f}(\gamma)=\int_{G} \overline{\gamma(g)} f(g) d \lambda(g) .
$$

For a subset $\Lambda$ of $\widehat{G}$, we define

$$
C_{\Lambda}(G)=\{f \in C(G): \hat{f}(\gamma)=0 \text { for all } \gamma \notin \Lambda\}
$$

and

$$
L_{\Lambda}^{\infty}(G)=\left\{f \in L^{\infty}(G): \hat{f}(\gamma)=0 \text { for all } \gamma \notin \Lambda\right\} .
$$

A subset $\Lambda$ of $\widehat{G}$ is called a Rosenthal set if $L_{\Lambda}^{\infty}(G)=C_{\Lambda}(G)$. A result of Lust-Piquard [5] says that $\Lambda$ is a Rosenthal set if and only if $C_{\Lambda}(G)$ has the Radon-Nikodym property.

For a complex Banach space $X$, we define

$$
L_{\Lambda}^{\infty}(G, X)=\left\{f \in L^{\infty}(G, X): \hat{f}(\gamma)=0 \text { for all } \gamma \notin \Lambda\right\},
$$


where $L^{\infty}(G, X)$ is the space of all (equivalence classes of) $X$-valued Bochner integrable functions defined on $G$ that are essentially bounded. $C_{\Lambda}(G, X)$ can be defined in a similar manner.

TheOREM. Let $G$ be a compact abelian metrizable group, let $\Lambda$ be a subset of $\widehat{G}$ and let $X$ be a complex Banach space. If $X$ has the RadonNikodym property and $L_{\Lambda}^{\infty}(G, X)$ is separable, then $L_{\Lambda}^{\infty}(G, X)$ has the Radon-Nikodym property.

Proof. To show that $L_{\Lambda}^{\infty}(G, X)$ has the Radon-Nikodym property it suffices to show that every bounded linear operator from $L^{1}[0,1]$ into $L_{\Lambda}^{\infty}(G, X)$ is Bochner representable [2]. So, let $T: L^{1}[0,1] \rightarrow L_{\Lambda}^{\infty}(G, X)$ be a bounded linear operator. For a Lebesgue measurable subset $A$ of $[0,1]$ and $B \in \mathscr{B}(G)$, define

$$
\mu(A \times B)=\int_{B} T\left(1_{A}\right)(g) d \lambda(g) .
$$

If we let $\mathscr{R}$ denote the algebra generated by sets of the form $A \times B$, where $A$ is a Lebesgue measurable subset of $[0,1]$ and $B \in \mathscr{B}(G)$, then it is easily shown that $\mu$ is a finitely additive $X$-valued measure on $\mathscr{R}$. Also,

$$
\begin{aligned}
\|\mu(A \times B)\| & \leq \int_{B}\left\|T\left(1_{A}\right)(g)\right\|_{X} d \lambda(g) \leq \int_{B}\left\|T\left(1_{A}\right)\right\|_{L_{\Lambda}^{\infty}(G, X)} d \lambda(g) \\
& =\lambda(B)\left\|T\left(1_{A}\right)\right\|_{L_{\Lambda}^{\infty}(G, X)} \\
& \leq \lambda(B)\|T\|\left\|1_{A}\right\|_{L^{1}[0,1]}=\lambda(B)\|T\| m(A) \\
& =\|T\|(m \times \lambda)(A \times B),
\end{aligned}
$$

where $m$ is Lebesgue measure on $[0,1]$. Thus $\|\mu(R)\| \leq\|T\|(m \times \lambda)(R)$ for all $R \in \mathscr{R}$.

If we let $\mathscr{A}$ denote the $\sigma$-algebra of Lebesgue measurable subsets of $[0,1]$, then the product $\sigma$-algebra, $\mathscr{A} \times \mathscr{B}(G)$, is generated by $\mathscr{R}$. Also, $m \times \lambda$ is a non-negative finite countably additive measure on $\mathscr{A} \times \mathscr{B}(G)$. For $E, F \in \mathscr{A} \times \mathscr{B}(G)$, define $d(E, F)=(m \times \lambda)(E \Delta F)$. Then $(\mathscr{A} \times \mathscr{B}(G), d)$ is a pseudo-metric space and $\mathscr{R}$ is dense in $\mathscr{A} \times \mathscr{B}(G), d)$. For $E$, $F \in \mathscr{R}, \mu(E)-\mu(F)=\mu(E \backslash F)-\mu(F \backslash E)$. Therefore,

$$
\begin{aligned}
\|\mu(E)-\mu(F)\| & \leq\|\mu(E \backslash F)\|+\|\mu(F \backslash E)\| \\
& \leq\|T\|(m \times \lambda)(E \backslash F)+\|T\|(m \times \lambda)(F \backslash E) \\
& =\|T\|(m \times \lambda)(E \Delta F) \\
& =\|T\| d(E, F) .
\end{aligned}
$$

Consequently, $\mu:(\mathscr{R}, d) \rightarrow(X,\|\cdot\|)$ is a Lipschitz function and since $(\mathscr{R}, d)$ is dense in $\mathscr{A} \times \mathscr{B}(G), d)$ there is a Lipschitz extension of $\mu$, 
$\bar{\mu}:(\mathscr{A} \times \mathscr{B}(G), d) \rightarrow(X,\|\cdot\|)$, such that $\bar{\mu}(R)=\mu(R)$ for all $R \in \mathscr{R}$. Thus, $\bar{\mu}$ is an $X$-valued countably additive measure on $\mathscr{A} \times \mathscr{B}(G)$ and $\|\bar{\mu}(S)\| \leq\|T\|(m \times \lambda)(S)$ for all $S \in \mathscr{A} \times \mathscr{B}(G)$. Hence, $\bar{\mu}$ is an $X$-valued measure of bounded average range and since $X$ has the Radon-Nikodym property there exists $F \in L^{\infty}([0,1] \times G, X)$ such that

$$
\bar{\mu}(S)=\int_{S} F(t, g) d(m \times \lambda)(t, g)
$$

for all $S \in \mathscr{A} \times \mathscr{B}(G)$.

In particular, for $A \in \mathscr{A}$ and $B \in \mathscr{B}(G)$,

$$
\begin{aligned}
\int_{B} T\left(1_{A}\right)(g) d \lambda(g) & =\mu(A \times B)=\bar{\mu}(A \times B)=\int_{A \times B} F(t, g) d(m \times \lambda)(t, g) \\
& =\int_{B} \int_{A} F(t, g) d m(t) d \lambda(g) .
\end{aligned}
$$

Now, for $x^{*} \in X^{*}$, define

$$
T_{x^{*}}: L^{1}[0,1] \rightarrow L_{\Lambda}^{\infty}(G) \quad \text { by }\left(T_{x^{*}} f\right)(g)=x^{*}((T f)(g))
$$

for $g \in G$ and $f \in L^{1}[0,1] . T_{x^{*}}$ is a bounded linear operator and $L_{\Lambda}^{\infty}(G)$ has the Radon-Nikodym property (this is so because $L_{\Lambda}^{\infty}(G)$ is a dual space which is separable since it is a subspace of the separable space $\left.L_{\Lambda}^{\infty}(G, X)\right)$. Therefore, there is $F_{x^{*}} \in L^{\infty}\left([0,1], L_{\Lambda}^{\infty}(G)\right)$ such that

$$
T_{x^{*}}(f)=\int_{[0,1]} f(t) F_{x^{*}}(t) d m(t)
$$

for all $f \in L^{1}[0,1]$. In particular, if $A \in \mathscr{A}$ and $g \in G$, then

$$
\begin{aligned}
x^{*}\left(T\left(1_{A}\right)(g)\right) & =\left(T_{x^{*}}\left(1_{A}\right)\right)(g)=\left[\int_{[0,1]} 1_{A}(t) F_{x^{*}}(t) d m(t)\right](g) \\
& =\int_{A}\left(F_{x^{*}}(t)\right)(g) d m(t) .
\end{aligned}
$$

Thus for $A \in \mathscr{A}, B \in \mathscr{B}(G)$ and $x^{*} \in X^{*}$

$$
\int_{B} x^{*}\left(T\left(1_{A}\right)(g)\right) d \lambda(g)=\int_{B} \int_{A}\left(F_{x^{*}}(t)\right)(g) d m(t) d \lambda(g) .
$$

Consequently, for all $A \in \mathscr{A}, B \in \mathscr{B}(G)$ and $x^{*} \in X^{*}$

$$
\begin{aligned}
\int_{B} \int_{A} x^{*}(F(t, g)) d m(t) d \lambda(g)=x^{*}\left[\int_{B} \int_{A} F(t, g) d m(t) d \lambda(g)\right] \\
\quad=x^{*}(\mu(A \times B)) \\
\quad=x^{*}\left[\int_{B} T\left(1_{A}\right)(g) d \lambda(g)\right]=\int_{B} x^{*}\left(T\left(1_{A}\right)(g) d \lambda(g)\right. \\
\quad=\int_{B} \int_{A}\left(F_{x^{*}}(t)\right)(g) d m(t) d \lambda(g) .
\end{aligned}
$$


The function $F_{x^{*}} \in L^{\infty}\left([0,1], L_{\Lambda}^{\infty}(G)\right)$, so by $[4$, p. 198], Theorem 17 , there exists $H_{x^{*}}:[0,1] \times G \rightarrow \mathbb{C}$ which is $m \times \lambda$-measurable and such that $F_{x^{*}}(t)=H_{x^{*}}(t, \cdot)$ for $m$-almost all $t \in[0,1]$. From this we get that for all $A \in \mathscr{A}$ and $B \in \mathscr{B}(G)$

$$
\int_{B} \int_{A} x^{*}(F(t, g)) d m(t) d \lambda(g)=\int_{B} \int_{A} H_{x^{*}}(t, g) d m(t) d \lambda(g) .
$$

Therefore $x^{*}(F(t, g))=H_{x^{*}}(t, g)$ for $m \times \lambda$-almost all $(t, g) \in[0,1] \times G$ (where the exceptional set of measure zero may vary with $\left.x^{*}\right)$. In particular, for $m$-almost all $t \in[0,1], x^{*}(F(t, g))=H_{x^{*}}(t, g)$ for $\lambda$-almost all $g \in$ $G$. This yields that for $m$-almost all $\left.t \in[0,1], x^{*}(F(t, g))=F_{x^{*}}(t)\right)(g)$ for $\lambda$-almost all $g \in G$. Therefore, $x^{*}(F(t, \cdot))$ is $\lambda$-measurable for $m$-almost all $t \in[0,1]$, where again we note that the exceptional set of measure zero may vary with $x^{*}$. However, since $X$ is separable, there is a countable norming set $\left\{x_{n}^{*}\right\}_{n=1}^{\infty}$ in the unit ball of $X^{*}$. From this we have that for all $n \in \mathbb{N}$, $x_{n}^{*}(F(t, \cdot)$ is $\lambda$-measurable for $m$-almost all $t \in[0,1]$. By Corollary 4 of $[2$, p. 42] we have that $F(t, \cdot)$ is $\lambda$-measurable for almost all $t \in[0,1]$.

$$
\text { Define } K(t)=\left\{\begin{array}{l}
F(t, \cdot) \text { if } F(t, \cdot) \text { is } \lambda \text {-measurable, } \\
0 \text { otherwise. }
\end{array}\right.
$$

For each $t \in[0,1], K(t): G \rightarrow X$ is $\lambda$-measurable and for $m$-almost all $t \in[0,1], x^{*}(K(t)(g))=x^{*}(F(t, g))=\left(F_{x^{*}}(t)\right)(g)$ for $\lambda$-almost all $g \in G$.

$$
\left\|F_{x_{n}^{*}}\right\|_{L^{\infty}\left([0,1], L_{\Lambda}^{\infty}(G)\right)}=\left\|T_{x_{n}^{*}}\right\| \leq\left\|x_{n^{*}}\right\|\|T\| \leq\|T\| .
$$

Therefore, for $m$-almost all $t \in[0,1]$ and for all $n \in \mathbb{N},\left\|F_{x_{n}^{*}}(t)\right\|_{L_{\Lambda}^{\infty}(G)} \leq$ $\|T\|$. Consequently, for $m$-almost all $t \in[0,1]$ and for all $n \in \mathbb{N}^{n},\left|F_{x_{n}^{*}}(t)(g)\right|$ $\leq\|T\|$ for $\lambda$-almost all $g \in G$. From this we get that for $m$-almost all $t \in[0,1]$ and for all $n \in \mathbb{N},\left|x_{n}^{*}(K(t)(g))\right| \leq\|T\|$ for $\lambda$-almost all $g \in G$. Since $\left\{x_{n}^{*}\right\}_{n=1}^{\infty}$ is a norming set we have that for $m$-almost all $t \in[0,1],\|K(t)(g)\|_{X} \leq\|T\|$ for $\lambda$-almost all $g \in G$. Thus, for $m$-almost all $t \in[0,1],\|K(t)\|_{L^{\infty}(G, X)} \leq\|T\|$.

Now define $K_{1}:[0,1] \rightarrow L^{\infty}(G, X)$ by

$$
K_{1}(t)=\left\{\begin{array}{l}
K(t) \text { if }\|K(t)\|_{L^{\infty}(G, X)} \leq\|T\|, \\
0 \text { otherwise. }
\end{array}\right.
$$

For $t \in[0,1], \gamma \notin \Lambda$ and $x^{*} \in X^{*}$

$$
x^{*}\left[\widehat{K_{1}(t)}(\gamma)\right]=\int_{G} x^{*}\left(K_{1}(t)(g)\right) \overline{\gamma(g)} d \lambda(g) .
$$

If $\|K(t)\|_{L^{\infty}(G, X)} \leq\|T\|$, then

$$
\begin{aligned}
x^{*}\left(\widehat{K_{1}(t)}(\gamma)\right) & \left.=\int_{G} x^{*}(K(t))(g)\right) \overline{\gamma(g)} d \lambda(g)=\int_{G}\left(F_{x^{*}}(t)\right)(g) \overline{\gamma(g)} d \lambda(g) \\
& =\widehat{F_{x^{*}}(t)}(\gamma)=0
\end{aligned}
$$


since $F_{x^{*}}(t) \in L_{\Lambda}^{\infty}(G)$. If $\|K(t)\|_{L^{\infty}(G, X)}>\|T\|$, then $x^{*}\left(\widehat{K_{1}(t)}(\gamma)\right)=0$. Hence $K_{1}:[0,1] \rightarrow L_{\Lambda}^{\infty}(G, X)$.

At this stage, we need to show $K_{1}$ is $m$-measurable. Since $L_{\Lambda}^{\infty}(G, X)$ is separable, it suffices to show that $K_{1}$ is scalarly measurable on a total set of linear functionals on $L_{\Lambda}^{\infty}(G, X)$ [1]. Note that for $\gamma \in \widehat{G}$ and $x^{*} \in X^{*}$, the map $x^{*} \otimes \gamma: L_{\Lambda}^{\infty}(G, X) \rightarrow \mathbb{C}$ defined by $\left(x^{*} \otimes \gamma\right)(f)=x^{*}(\widehat{f}(\cdot))(\gamma)$ is a bounded linear functional. The total set we will use is the set $\left\{x_{n}^{*} \otimes \gamma\right.$ : $n \in \mathbb{N}, \gamma \in \Lambda\}$ where $\left\{x_{n}^{*}\right\}$ is a norming set in $X^{*}$. Note that this set is countable since $\Lambda$ is countable.

For $n \in \mathbb{N}$ and $\gamma \in \Lambda$,

$$
\left(x_{n}^{*} \otimes \gamma\right)\left(K_{1}(t)\right)=\int_{G} x_{n}^{*}\left(K_{1}(t)(g)\right) \overline{\gamma(g)} d \lambda(g)=\int_{G} x_{n}^{*}(F(t, g)) \overline{\gamma(g)} d \lambda(g) .
$$

However, since $x_{n}^{*}(F(t, g))$ is a measurable function of $t$, so is the above integral and hence $\left(x_{n}^{*} \otimes \gamma\right)\left(K_{1}(t)\right)$ is a measurable function of $t$. Therefore, $K_{1}$ is an $m$-measurable function.

To complete the proof we must show that for all $A \in \mathscr{A}, T\left(1_{A}\right)=$ $\int_{A} K_{1}(t) d m(t)$. For $A \in \mathscr{A}$ and $B \in \mathscr{B}(G)$,

$\int_{B} T\left(1_{A}\right)(g) d \lambda(g)=\int_{B} \int_{A} F(s, t) d m(t) d \lambda(g)=\int_{B} \int_{A} K_{1}(t)(g) d m(t) d \lambda(g)$.

Thus, for $\lambda$-almost all $g \in G$,

$$
T\left(1_{A}\right)(g)=\int_{A} K_{1}(t)(g) d m(t)
$$

and so as elements of $L_{\lambda}^{\infty}(G, X), T\left(1_{A}\right)=\int_{A} K_{1}(t) d m(t)$. Hence $L_{\Lambda}^{\infty}(G, X)$ has the Radon-Nikodym property.

Remark. The converse of the theorem is also true: if $L_{\Lambda}^{\infty}(G, X)$ has the Radon-Nikodym property and $X$ is separable, then $L_{\Lambda}^{\infty}(G, X)$ is separable. The proof can essentially be found in [5].

COROLlary 1. Let $G$ be a compact abelian metrizable group, $\Lambda$ a subset of $\widehat{G}$ and $X$ a complex Banach space with the Radon-Nikodym property. If $\mathscr{L}\left(L^{1}(G) / L_{\Lambda^{\prime}}^{1}(G), X\right)$ is separable, then it has the Radon-Nikodym property $\left(\Lambda^{\prime}=\{\gamma: \bar{\gamma} \notin \Lambda\}\right)$.

Proof. Since $X$ has the Radon-Nikodym property, $\mathscr{L}\left(L^{1}(G) / L_{\Lambda^{\prime}}^{1}(G), X\right)$ is isometrically isomorphic to $L_{\Lambda}^{\infty}(G, X)[2$, p. 63]. Now apply the Theorem.

REMARK. A close analysis of the proof of Theorem 1 allows one to generalise Corollary 1 to obtain the following: 
Let $(\Omega, \Sigma, \mu)$ be a probability space such that $L^{1}(\mu)$ is separable. Let $Y$ be a closed subspace of $L^{1}(\mu)$. If $X$ is a Banach space with the RadonNikodym property and if $\mathscr{L}\left(L^{1}(\mu) / Y, X\right)$ is separable, then $\mathscr{L}\left(L^{1}(\mu) / Y, X\right)$ has the Radon-Nikodym property.

COROLlary 2. Let $G$ be a compact abelian metrizable group, let $\Lambda$ be a subset of $\widehat{G}$ and let $X$ be a complex Banach space. If $\Lambda$ is a Rosenthal set and $X$ has the Radon-Nikodym property and the Schur property, then $C_{\Lambda}(G, X)$ has the Radon-Nikodym property.

Proof. It suffices to show that every separable subspace of $C_{\Lambda}(G, X)$ has the Radon-Nikodym property. Notice that each separable subspace of $C_{\Lambda}(G, X)$ is a subspace of $C_{\Lambda}(G, Y)$ for some separable subspace $Y$ of $X$. We will be finished if we can show that $C_{\Lambda}(G, Y)$ has the Radon-Nikodym property for every separable subspace $Y$ of $X$.

Let $Y$ be a separable subspace of $X$. We will show that $C_{\Lambda}(G, Y)$ is isomorphic to $L_{\Lambda}^{\infty}(G, Y)$. By Theorem 5 of $\left[2\right.$, p. 63], $L_{\Lambda}^{\infty}(G, Y)$ is isomorphic to $\mathscr{L}\left(L^{1}(G) / L_{\Lambda^{\prime}}(G), Y\right)$. However, since $L_{\Lambda}^{\infty}(G)$ is a separable dual, $L^{1}(G) / L_{\Lambda^{\prime}}^{1}(G)$ does not contain a copy of $\ell^{1}$. Hence every bounded linear operator from $L^{1}(G) / L_{\Lambda^{\prime}}^{1}(G)$ to $Y$ is compact because $Y$ has the Schur property. $L_{\Lambda}^{\infty}(G)$ has the approximation property so the space of all compact operators from $L^{1}(G) / L_{\Lambda^{\prime}}^{1}(G)$ to $Y$ is isomorphic to $L_{\Lambda}^{\infty}(G) \ddot{\otimes} Y$. But $L_{\Lambda}^{\infty}(G) \ddot{\otimes} Y=C_{\Lambda}(G) \ddot{\otimes} Y$, which in turn is isomorphic to $C_{\Lambda}(G, Y)$. Thus we have shown that $C_{\Lambda}(G, Y)$ is isomorphic to $L_{\Lambda}^{\infty}(G, Y) . C_{\Lambda}(G, Y)$ is separable since $Y$ is separable and so $L_{\Lambda}^{\infty}(G, Y)$ has the Radon-Nikodym property by the Theorem. Hence $C_{\Lambda}(G, Y)$ has the Radon-Nikodym property and so the proof is complete.

ReMARX. In [6], Lust-Piquard shows that if $G$ is a compact abelian metrizable group and $\Lambda$ is a subset of $\widehat{G}$ is such that $L_{\Lambda}^{\infty}(G)$ has the Schur property, then $\Lambda$ is a Rosenthal set.

COROLlaRY 3. Let $G_{1}$ and $G_{2}$ be two compact abelian metrizable groups and let $\Lambda_{1}$ and $\Lambda_{2}$ be subsets of $\widehat{G}_{1}$ and $\widehat{G}_{2}$, respectively. If $\Lambda_{1}$ is a Rosenthal set and $L_{\Lambda_{2}}^{\infty}\left(G_{2}\right)$ has the Schur property, then $\Lambda_{1} \times \Lambda_{2}$ is a Rosenthal subset of $\widehat{G}_{1} \times \widehat{G}_{2}$.

Proof. To show that $\Lambda_{1} \times \Lambda_{2}$ is a Rosenthal set, it suffices to show that $C_{\Lambda_{1} \times \Lambda_{2}}\left(G_{1} \times G_{2}\right)$ has the Radon-Nikodym property [5]. $C_{\Lambda_{1} \times \Lambda_{2}}\left(G_{1} \times G_{2}\right)$ is isomorphic to $C_{\Lambda_{1}}\left(G_{1}, C_{\Lambda_{2}}\left(G_{2}\right)\right)$. Since $L_{\Lambda_{2}}^{\infty}\left(G_{2}\right)$ has the Schur property, 
$\Lambda_{2}$ is a Rosenthal set, by the preceding Remark, so $C_{\Lambda_{2}}\left(G_{2}\right)$ has the RadonNikodym property. An application of Corollary 2 completes the proof.

REMARK. Corollary 3 was proved independently by F. Lust-Piquard in [8, Chapter 4, Theorem 5].

\section{Acknowledgement}

The author wishes to thank the referee for many insightful comments and suggestions and for providing him a copy of [8].

\section{References}

[1] A. V. Bukhvalov, 'Radon-Nikodym property in Banach spaces of measurable vectorvalued functions', Mat. Zametki 26 (1979), 875-884. English translation: Math. Notes 26 (1979), 939-944.

[2] J. Diestel and J. J. Uhl, Jr., Vector Measures, Math. Surveys Monographs, 15 (Amer. Math. Soc., Providence, 1977).

[3] J. Diestel and J. J. Uhl, Jr., 'Progress in vector measures-1977-1983', in: Measure Theory and its Applications, Lecture Notes in Mathematics, 1033 (Springer, Berlin, 1983), pp. 144-192.

[4] N. Dunford and J. T. Schwartz, Linear operators, Part I (Interscience Publishers, New York, 1957).

[5] F. Lust-Piquard, 'Ensenbles de Rosenthal et ensembles de Riesz', C. R. Acad. Sci. Paris Sér. I Math. 282 (1976), 833-835.

[6] F. Lust-Piquard, 'L'espace des fonctions presque-periodiques dont le spectre est contenu dans un ensemble compact denombrable á la propriete de Schur', Colloq. Math. 41 (1979), 273-284.

[7] F. Lust-Piquard, 'Propriétés geometriques des sous-espaces invariants par translation de $L^{1}(G)$ et $C(G)$ ', Séminaire sur la geometrie des espaces de Banach, Ecole Polytechnique (1977-78), Exposé 26.

[8] F. Lust-Piquard, 'Propriétés harmoniques et geometriques des sous-espaces invariants par translation de $L^{\infty}(G)$ ', (Ph.D. Thesis, Université Paris-Sud, 1978).

Miami University

Oxford, Ohio 45056

U.S.A. 